\title{
Collisions of oppositely charged black holes
}

\author{
Miguel Zilhão, ${ }^{1, *}$ Vitor Cardoso, ${ }^{2,3}$ Carlos Herdeiro, ${ }^{4}$ Luis Lehner, ${ }^{3}$ and Ulrich Sperhake ${ }^{5,6,7}$ \\ ${ }^{1}$ Center for Computational Relativity and Gravitation and School of Mathematical Sciences, \\ Rochester Institute of Technology, Rochester, New York 14623, USA \\ ${ }^{2}$ CENTRA, Departamento de Física, Instituto Superior Técnico, Universidade de Lisboa, \\ Avenida Rovisco Pais 1, 1049 Lisboa, Portugal \\ ${ }^{3}$ Perimeter Institute for Theoretical Physics, Waterloo, Ontario N2L 2Y5, Canada \\ ${ }^{4}$ Departamento de Física da Universidade de Aveiro and I3N, \\ Campus de Santiago, 3810-183 Aveiro, Portugal \\ ${ }^{5}$ Department of Applied Mathematics and Theoretical Physics, Centre for Mathematical Sciences, \\ University of Cambridge, Cambridge CB3 OWA, United Kingdom \\ ${ }^{6}$ Theoretical Astrophysics 350-17, California Institute of Technology, Pasadena, California 91125, USA \\ ${ }^{7}$ Department of Physics and Astronomy, The University of Mississippi, University, Mississippi 38677, USA
}

(Received 3 December 2013; published 11 February 2014)

The first fully nonlinear numerical simulations of colliding charged black holes in $D=4$ EinsteinMaxwell theory were recently reported [Zilhão et al., Phys. Rev. D 85, 124062 (2012)]. These collisions were performed for black holes with equal charge-to-mass ratio, for which initial data can be found in closed analytic form. Here we generalize the study of collisions of charged black holes to the case of unequal charge-to-mass ratios. We focus on oppositely charged black holes, as to maximize accelerationdependent effects. As $|Q| / M$ increases from 0 to 0.99 , we observe that the gravitational radiation emitted increases by a factor of $\sim 2.7$; the electromagnetic radiation emission becomes dominant for $|Q| / M \gtrsim 0.37$ and at $|Q| / M=0.99$ is larger, by a factor of $\sim 5.8$, than its gravitational counterpart. We observe that these numerical results exhibit a precise and simple scaling with the charge. Furthermore, we show that the results from the numerical simulations are qualitatively captured by a simple analytic model that computes the electromagnetic dipolar radiation and the gravitational quadrupolar radiation of two nonrelativistic interacting particles in Minkowski spacetime.

DOI: $10.1103 /$ PhysRevD.89.044008

PACS numbers: 04.25.D-, 04.25.dg, 04.40.Nr

\section{INTRODUCTION}

Astrophysical black hole (BH) collisions are expected to release tremendous amounts of energy through gravitational waves. For instance, a binary system of two nonspinning equal mass $\mathrm{BHs}$ is expected to release over $3 \%$ of its total energy into gravitational radiation, during the plunge/merger phase [1]. This amounts to a peak luminosity of about $10^{56} \mathrm{erg} / \mathrm{s}$, i.e., $\sim 10^{23} L_{\odot}$. One may then ask: how large can the efficiency of any BH collision be, in converting the energy of the BHs into radiation?

The simplest argument that bounds this efficiency was provided by Hawking and is based on the area theorem [2]. The second law of thermodynamics indicates that for the (head-on) collision of two Schwarzschild BHs starting from rest, no more than $29 \%$ of the initial energy contained in the $\mathrm{BHs}$ can be converted into gravitational radiation. For BHs colliding head-on at very high energies, on the other hand, a completely different argument originally due to Penrose [3], based on the existence of an apparent horizon in the head-on collision of shock waves, curiously yields exactly the same bound of 29\% (see [4] for its $D$-dimensional generalization). The former estimate turns out to be an

*mzilhao@astro.rit.edu extremely conservative limit, as numerical relativity simulations of BH collisions show that for BHs starting from rest the energy released is much lower-of the order of $0.05 \%$ of the total energy [5]. On the other hand, the latter estimate is only off by a factor of $\simeq 2$, as the energy released for high energy collisions approaches $14 \%$ in the ultrarelativistic limit [6], a mismatch by merely a factor of 2 with the shock waves estimate.

The efficiency in converting the energy of a system of $\mathrm{BHs}$ into radiation can be further changed by introducing impact parameter and spin. Collisions from rest of $\mathrm{BHs}$ with aligned spins perpendicular to the collision axis radiate $0.118 \%$ of the total energy when $a / M \simeq 0.4$ [7], while for the antialigned case a lower radiation efficiency of $0.090 \%$ has been found for $a / M \simeq 0.75$ [8]. For $\mathrm{BH}$ binaries in quasicircular orbits, spins aligned with the orbital angular momentum significantly increase the amount of energy radiated in gravitational waves [9]. Extrapolated to the extremal limit $a / M=1$, the prediction is $E_{\mathrm{rad}} / M=11.40 \%$ [10]. A more recent analysis [11] has further led to the following two conclusions: (i) for high energy collisions of spinning BHs with an impact parameter, in the ultrarelativistic limit, about $50 \%$ of the energy can be radiated away; (ii) for $v / c \gtrsim 0.9$ spin effects become 
washed away. This latter observation serves to support the idea that matter does not matter at very high energies, since processes should be dominated by the kinetic energy and hence details of the internal structure of the colliding objects, as, for example, spin or charge, should become irrelevant. Earlier evidence for this hypothesis had already been provided by high energy collisions of boson fields and fluid particles [12-14]. The former observation, on the other hand, finds a curious parallel in higher dimensional head-on collisions of shock waves: both, apparent horizon arguments [4] and perturbative analysis [15,16], suggest that the radiative efficiency is always smaller than 50\% approaching this value in the limit of an infinite number of dimensions.

The profound influence that the additional physical parameters discussed above (spin and impact parameter) have in the total radiated energy prompts the question of whether charges can also affect the outcome. Do collisions of charged BHs support these two observations? That is, can no more than $50 \%$ of the energy be radiated away in any $\mathrm{BH}$ collision and the details of the $\mathrm{BH}$ structurenamely its charge - become irrelevant at very high energies? In particular, concerning the first point, Hawking's area theorem argument suggests that the analysis of charged BHs may be of special relevance, as a simple comparison with the rotating case reveals. In a head-on collision of equal mass $M$, antialigned spins $\pm a M$ Kerr $\mathrm{BHs},{ }^{1}$ starting from rest, the fraction of radiated energy is bounded by

$$
\epsilon \leq 1-\frac{1}{2} \sqrt{1+\sqrt{1-(a / M)^{2}}}
$$

which varies from $29 \%$ to $50 \%$ as $|a|$ varies from zero to $M$. On the other hand, in a head-on collision of equal mass $M$, opposite charge $\pm Q$ Reissner-Nordström $\mathrm{BHs}$, starting from rest, the fraction of radiated energy is bounded by

$$
\epsilon \leq 1-\frac{1}{2 \sqrt{2}}\left(1+\sqrt{1-(Q / M)^{2}}\right),
$$

which varies from $29 \%$ to $65 \%$ as $|Q|$ varies from zero to $M$. In spite of this argument providing a poor estimate of the actual value, it indicates a larger increase in $E_{\mathrm{rad}}$ if the maximum amount of charge is added to each $\mathrm{BH}$ in comparison with adding instead the maximum spin. High energy collisions of oppositely charged $\mathrm{BHs}$ provide particularly interesting problems to test the aforementioned observations.

The first step toward this goal was taken in [18], hereafter referred to as Paper I, where collisions of charged

\footnotetext{
${ }^{1}$ To have a head-on collision of Kerr BHs starting from rest, their spins must be either aligned or antialigned; to maximize accelerations and hence the emitted radiation we choose antialigned spins, since the spin-spin force becomes attractive [17].
}

BHs in Einstein-Maxwell theory were studied. That work was restricted to the case of head-on collisions from rest of BHs with equal charge-to-mass ratios, which admits analytic initial data. In this paper, an important extension is given as we study collisions of charged BHs with unequal charge-to-mass ratios; in particular, we consider oppositely charged BHs in view of the above motivations. These configurations require a numerical construction of initial data. We generate such initial data using a modified version of the TwOPUNCTURES spectral solver [19] that is described in Sec. II along with a summary of the formulation of the Einstein-Maxwell equations used in our code. In Sec. III we produce analytic estimates based on considerations of point charges and masses in flat space using electromagnetic dipolar emission and quadrupolar gravitational emission approximations. We show that these estimates can reproduce qualitatively-and for an appropriate value of a cutoff parameter even quantitatively - the results of the numerical simulations. The fully nonlinear numerical simulations in Einstein-Maxwell theory are presented in Sec. IV where, in particular, a simple and precise scaling of the waveforms and of the radiated energy with the charge is observed. Concluding remarks are presented in Sec. V.

\section{FORMALISM}

As in Paper I, we shall consider the enlarged electrovacuum Einstein-Maxwell equations,

$$
\begin{aligned}
R_{\mu \nu}-\frac{R}{2} g_{\mu \nu} & =8 \pi T_{\mu \nu}, \\
\nabla_{\mu}\left(F^{\mu \nu}+g^{\mu \nu} \Psi\right) & =-\kappa n^{\nu} \Psi, \\
\nabla_{\mu}\left(\star F^{\mu \nu}+g^{\mu \nu} \Phi\right) & =-\kappa n^{\nu} \Phi,
\end{aligned}
$$

where $F^{\mu \nu}$ is the Maxwell tensor and $\star F^{\mu \nu}$ its Hodge dual, $\kappa$ is a constant, and $n^{\mu}$ is the 4-velocity of the Eulerian observer. We recover the standard Einstein-Maxwell system when $\Psi=0=\Phi$. With the scalar field $\Psi$ and pseudoscalar $\Phi$ introduced in this way, the evolution of this system drives $\Psi$ and $\Phi$ to zero (for positive $\kappa$ ), thus ensuring the magnetic and electric constraints are controlled [20,21]. The electromagnetic stress-energy tensor takes the usual form

$$
T_{\mu \nu}=\frac{1}{4 \pi}\left[F_{\mu}{ }^{\lambda} F_{\nu \lambda}-\frac{1}{4} g_{\mu \nu} F^{\lambda \sigma} F_{\lambda \sigma}\right] .
$$

The $3+1$ decomposition was detailed in Paper I. Here we recall that we introduce a 3-metric

$$
\gamma_{\mu \nu}=g_{\mu \nu}+n_{\mu} n_{\nu}
$$

and we denote by $D_{i}$ the covariant derivative associated with $\gamma_{i j}$, where $i, j=1,2,3$ are spatial indices. The Maxwell tensor and its dual are decomposed in the electric and magnetic 4-vectors as 


$$
\begin{aligned}
F_{\mu \nu} & =n_{\mu} E_{\nu}-n_{\nu} E_{\mu}+\epsilon_{\mu \nu \alpha \beta} B^{\alpha} n^{\beta}, \\
\star F_{\mu \nu} & =n_{\mu} B_{\nu}-n_{\nu} B_{\mu}-\epsilon_{\mu \nu \alpha \beta} E^{\alpha} n^{\beta},
\end{aligned}
$$

where we use the convention $\epsilon_{1230}=\sqrt{-g}, \quad \epsilon_{\alpha \beta \gamma}=$ $\epsilon_{\alpha \beta \gamma \delta} n^{\delta}, \epsilon_{123}=\sqrt{\gamma}$.

In Paper I, BH binaries with equal charge and mass colliding from rest were considered. Such configurations allow for initial data to be specified in fully analytical form using the Brill-Lindquist construction [22]. We here want to consider $\mathrm{BH}$ binaries with different charge-to-mass ratios, which no longer admit this simple construction. We will thus follow the procedure presented in [23], which we outline in the following.

Assuming time-symmetric initial configurations, i.e., such that the extrinsic curvature vanishes, $K_{i j}=0$, combined with the condition of an initially vanishing magnetic field, the magnetic constraint $D_{i} B^{i}=0$, and momentum constraint are automatically satisfied. By further assuming the spatial metric to be conformally flat

$$
\gamma_{i j} d x^{i} d x^{j}=\psi^{4}\left(d x^{2}+d y^{2}+d z^{2}\right)
$$

the Hamiltonian constraint reduces to

$$
\triangle \psi+\frac{1}{4} \psi^{9} E^{i} E^{j} \delta_{i j}=0,
$$

where $\triangle$ is the flat space Laplace operator. The electric constraint, Gauss's law, has the usual form

$$
D_{i} E^{i}=0
$$

and can be solved independently of (2.6). Introducing an electric potential $\varphi$ through

$$
E^{i}=-\psi^{-6} \delta^{i j} \partial_{j} \varphi,
$$

we find that

$$
\varphi=\sum_{i=1}^{N} \frac{q_{i}}{\left|\mathbf{x}-\mathbf{x}_{i}\right|},
$$

where $\mathbf{x}_{i} \equiv\left(x_{i}, y_{i}, z_{i}\right)$ is the coordinate location of the $i$ th "puncture," solves (2.7). Equation (2.6) then takes the form

$$
\triangle \psi+\frac{1}{4} \psi^{-3} \partial_{i} \varphi \partial_{j} \varphi \delta^{i j}=0 .
$$

Following [23], we now assume the following ansatz for $\psi$ :

$$
\psi^{2}=(u+\eta)^{2}-\frac{\varphi^{2}}{4},
$$

where

$$
\eta=\sum_{i=1}^{N} \frac{m_{i}}{2\left|\mathbf{x}-\mathbf{x}_{i}\right|}
$$

Equation (2.10), in terms of the new variable $u$, then reads

$$
\begin{aligned}
\triangle u & -\frac{\varphi^{2}}{4 \psi^{2}(u+\eta)}\left(\partial_{k} u \partial^{k} u+2 \partial_{k} u \partial^{k} \eta+\partial_{k} \eta \partial^{k} \eta\right) \\
& +\frac{\varphi}{2 \psi^{2}}\left(\partial_{k} u \partial^{k} \varphi+\partial_{k} \eta \partial^{k} \varphi\right) \\
& +\frac{1-(u+\eta)^{2}}{4(u+\eta) \psi^{2}} \partial_{k} \varphi \partial^{k} \varphi=0
\end{aligned}
$$

where $\partial^{k} \equiv \delta^{k l} \partial_{l}$. Note that when choosing configurations of BHs with the same charge-to-mass ratio, Eq. (2.13) is immediately solved with $u=1$, and we recover the cases studied in Paper I.

For our present purposes, we fix $m_{1}=m_{2} \equiv M / 2$, $q_{1}=-q_{2} \equiv Q / 2$, and $z_{1}=-z_{2} \equiv d / 2$, and solve (2.13) by adapting the spectral solver TwoPunctures [19]. Originally developed to calculate four-dimensional vacuum puncture data corresponding to both single and binary $\mathrm{BH}$ configurations, TwoPUNCTURES has been successfully adapted in the past to tackle different configurations (such as higher-dimensional puncture data [24]). We here take a pragmatic approach to the initial data solving, and we merely modify the relevant source terms of the TwoPunCTURES routines according to (2.13).

As noted in [23], the function $u$ to solve for turns out to be only $C^{0}$ at the punctures, so the exponential convergence properties of the TwoPunctures solver are lost. This, however, is no concern because in practice the numerical constraint violations are dominated by the discretization errors accumulated in the time evolution such that this relatively minor decrease in the accuracy of the initial data is not noticeable in the evolution. Furthermore, we have cross-checked our initial data for several examples with those obtained by the authors of [23] and found very good agreement. $^{2}$

The main physical observables we shall be interested in are the electromagnetic and gravitational radiation emitted in the collision process. To extract the radiation components we again follow the procedure described in Paper I. For the gravitational wave signal we calculate the NewmanPenrose scalar $\Psi_{4}$ defined as

$$
\Psi_{4} \equiv C_{\alpha \beta \gamma \delta} k^{\alpha} \bar{m}^{\beta} k^{\gamma} \bar{m}^{\delta},
$$

where $C_{\alpha \beta \gamma \delta}$ is the Weyl tensor and $k, \bar{m}$ are part of a null tetrad $l, k, m, \bar{m}$ satisfying $-l \cdot k=1=m \cdot \bar{m}$; all other inner products vanish. For analyzing the behavior of the electromagnetic fields we compute the scalar functions $\Phi_{1}$ and $\Phi_{2}$ [25], defined as

\footnotetext{
${ }^{2}$ We thank J. C. Degollado for these comparisons.
} 


$$
\begin{gathered}
\Phi_{1} \equiv \frac{1}{2} F_{\mu \nu}\left(l^{\mu} k^{\nu}+\bar{m}^{\mu} m^{\nu}\right), \\
\Phi_{2} \equiv F_{\mu \nu} \bar{m}^{\mu} k^{\nu} .
\end{gathered}
$$

For outgoing waves at infinity, the relevant scalar behaves as

$$
\Phi_{2} \sim E_{\hat{\theta}}-i E_{\hat{\phi}} .
$$

For static charges, the scalars behave as

$$
\Phi_{1} \sim \frac{1}{2} E_{\hat{r}}, \quad \Phi_{2} \sim \frac{1}{2}\left(E_{\hat{\theta}}-i E_{\hat{\phi}}\right) .
$$

At a given extraction radius $R_{\mathrm{ex}}$, we perform a multipolar decomposition by projecting $\Psi_{4}, \Phi_{1}$, and $\Phi_{2}$ onto spherical harmonics of spin weight $s=-2,0$, and -1 , respectively,

$$
\begin{aligned}
& \Psi_{4}(t, \theta, \phi)=\sum_{l, m} \psi^{l m}(t) Y_{l m}^{-2}(\theta, \phi), \\
& \Phi_{1}(t, \theta, \phi)=\sum_{l, m} \phi_{1}^{l m}(t) Y_{l m}^{0}(\theta, \phi), \\
& \Phi_{2}(t, \theta, \phi)=\sum_{l, m} \phi_{2}^{l m}(t) Y_{l m}^{-1}(\theta, \phi) .
\end{aligned}
$$

In terms of these multipoles, the radiated flux and energy are given by the expressions [25]

$$
\begin{gathered}
P_{\mathrm{GW}}=\frac{d E_{\mathrm{GW}}}{d t}=\lim _{r \rightarrow \infty} \frac{r^{2}}{16 \pi} \sum_{l, m}\left|\int_{-\infty}^{t} d t^{\prime} \psi^{l m}\left(t^{\prime}\right)\right|^{2}, \\
P_{\mathrm{EM}}=\frac{d E_{\mathrm{EM}}}{d t}=\lim _{r \rightarrow \infty} \frac{r^{2}}{4 \pi} \sum_{l, m}\left|\phi_{2}^{l m}(t)\right|^{2} .
\end{gathered}
$$

\section{ANALYTIC PREDICTIONS}

Before presenting the results of our numerical simulations, we will discuss a simple analytic approximation to gain an intuitive understanding of the binary's dynamics. This analysis also provides predictions to compare with the numerical results presented below.

Consider the electrodynamics of a system of two oppositely charged point charges in a Minkowski background spacetime. As in the $\mathrm{BH}$ case, we denote by $q_{1}=$ $-q_{2} \equiv Q / 2$ and $m_{1}=m_{2} \equiv M / 2$ the electric charge and mass of the particles that are initially at rest at position $z= \pm d / 2$. The expected behavior of the radial component of the resulting electric field is given by [26]

$$
E_{\hat{r}}=4 \pi \sum_{l=0}^{\infty} \sum_{m=-l}^{l} \frac{l+1}{2 l+1} q_{l m} \frac{Y_{l m}(\theta, \varphi)}{r^{l+2}},
$$

where $q_{l m}=\int Y_{l m}\left(\theta^{\prime}, \phi^{\prime}\right)^{*}\left(r^{\prime}\right)^{l} \rho\left(\mathbf{x}^{\prime}\right) d^{3} \mathbf{x}^{\prime}$ are the multipole moments and $\rho$ is the charge density. The leading term of this multipolar expansion for our system of two opposite charges is the dipole

$$
E_{\hat{r}} \simeq \sqrt{\frac{4 \pi}{3}} Q d \frac{Y_{10}}{r^{3}}
$$

whereas the monopole term vanishes because the total charge is zero. In a similar fashion, the $\theta$ component of the electric field becomes

$$
E_{\hat{\theta}} \simeq-\sqrt{\frac{2 \pi}{3}} Q d \frac{Y_{10}^{-1}}{r^{3}} .
$$

An estimate for the dipole amplitude in the limit of two static point charges is then obtained from inserting the radial and poloidal components of the electric field (3.2) and (3.3) into the expressions (2.18) for $\Phi_{1}$ and $\Phi_{2}$ and its multipolar decompositions (2.20) and (2.21)

$$
\begin{gathered}
r^{3} \phi_{1}^{10}=\sqrt{\frac{\pi}{3}} Q d \approx 1.02333 Q d, \\
r^{3} \phi_{2}^{10}=-\sqrt{\frac{\pi}{6}} Q d \approx-0.72360 Q d .
\end{gathered}
$$

Although the actual setup is dynamical, one expects this expression to provide a reasonably good approximation in the initial stages of the numerical evolution. The comparison between this approximation and the numerical simulations will be performed below in Fig. 1. After the merger and ringdown the dipole will eventually approach zero as a single merged $\mathrm{BH}$ corresponds to the case $d=0$ in Eqs. (3.4) and (3.5).

Let us now follow the nonrelativistic dynamics of the two charges in Minkowski spacetime assuming their interaction is encoded in the Newtonian gravitational energy plus the electrostatic energy:

$$
V=-\frac{G M^{2}}{4 d}-\frac{1}{16 \pi \varepsilon_{0}} \frac{Q^{2}}{d} .
$$

Our aim is to obtain the radiated energy in the collision using dipole/quadrupole emission formulas for electromagnetic/gravitational radiation. This is expected to be a good approximation for systems where the accelerations involved are not too large and it has yielded good estimates in the case of equal charges, as shown in Paper I. Using units with $G=4 \pi \varepsilon_{0}=1$, conservation of energy implies that under their mutual attraction the motion along the $z$ axis obeys

$$
M \dot{z}^{2}-\frac{M^{2} \mathcal{B}}{4 z}=-\frac{M^{2} \mathcal{B}}{2 d},
$$



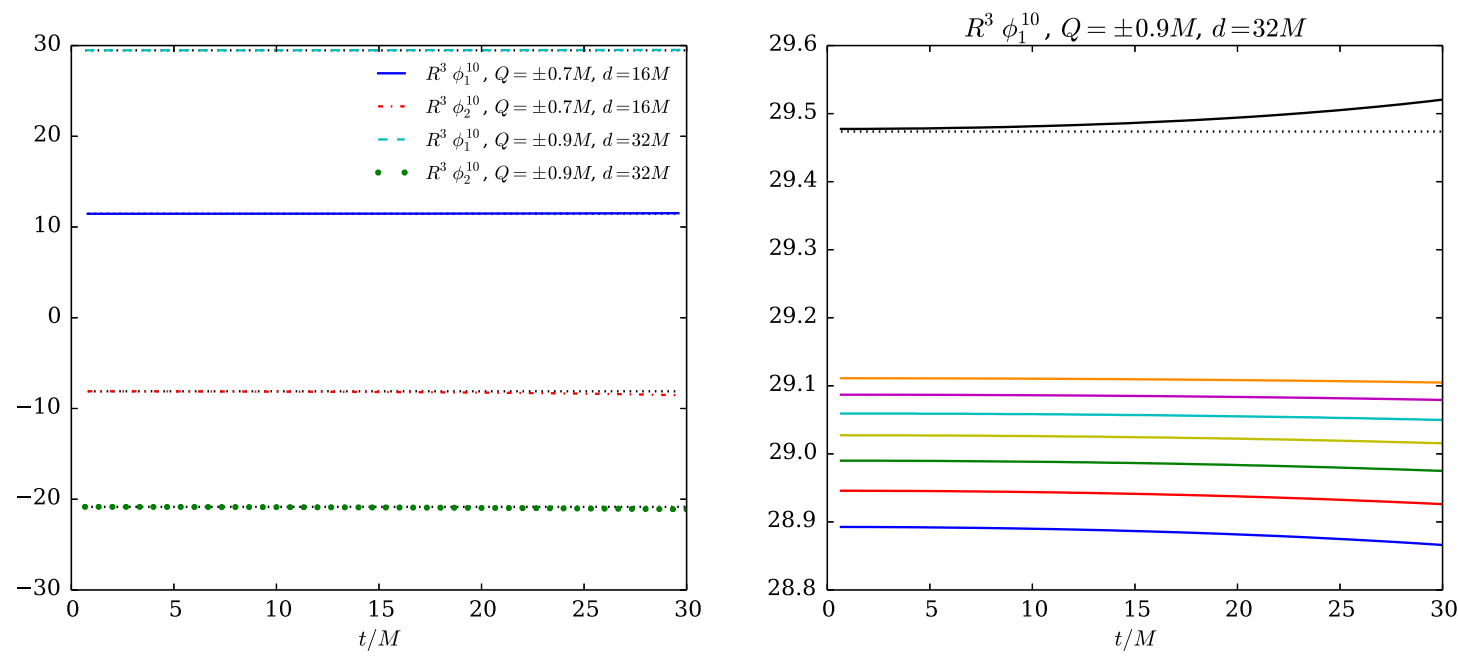

FIG. 1 (color online). Predictions from Eqs. (3.4) and (3.5) (black dotted lines) matched against our simulation results (models Q+070_D16_HF80 and Q+-090_D32_HF192; see Table I). Left plot shows results obtained from fitting a curve of the form $R_{\mathrm{ex}}^{3} \phi_{1,2}^{10}=$ $a_{0}+a_{1} / R_{\text {ex }}$ to the numerically extracted $\phi_{1,2}^{10}$ (for all time steps) using all available extraction radii. Right plot (showing only the $Q= \pm 0.9 M$ case) depicts explicitly the extracted $R_{\mathrm{ex}}^{3} \phi_{1}^{10}$ for all extraction radii. In this case, the curves from bottom to top correspond to $R_{\mathrm{ex}}=100 M, \ldots, 160 M$ in steps of $10 M$, with the uppermost being the result extrapolated to infinity. We note that for these cases, the BH merger happens roughly at around $t \sim 330 M$.

where

$$
\mathcal{B} \equiv 1+Q^{2} / M^{2}
$$

The resulting equation of motion for $z(t)$ is obtained by differentiating Eq. (3.7), which results in

$$
M \ddot{z}=-\frac{M^{2}}{8 z^{2}}-\frac{Q^{2}}{8 z^{2}}=-M^{2} \frac{\mathcal{B}}{8 z^{2}} .
$$

To compare the emission of gravitational and electromagnetic radiation we use the quadrupole formula for the total power emitted in gravitational radiation,

$$
P_{\mathrm{GW}}=\frac{G}{45 c^{5}} \sum_{i j}\left(\bar{Q}_{i j}\right)^{2},
$$

where the (traceless) quadrupole tensor $Q_{i j}=$ $\int d^{3} \mathbf{x} \rho_{m}(\mathbf{x})\left(3 x_{i} x_{j}-r^{2} \delta_{i j}\right)$, where $\rho_{m}$ is the matter energy density, and the Larmor dipole formula for the total power emitted in electromagnetic radiation by a time-varying dipole [26]

$$
P_{\mathrm{EM}}=\frac{1}{6 \pi \varepsilon_{0} c^{3}} \ddot{\mathbf{d}}^{2},
$$

where $\mathbf{d}$ is the dipole vector that has components $d_{i}=\int d^{3} \mathbf{x} \rho_{e}(\mathbf{x}) x_{i}$, and $\rho_{e}$ is the electric charge density. For clarity we have reinstated the factors $G, c$, and $4 \pi \varepsilon_{0}$ in the last two formulas. We shall drop them in the following. To compute (3.10) and (3.11) we use (3.7) and (3.9) to find

$$
P_{\mathrm{GW}}=\frac{\mathcal{B}^{3} M^{5}}{480 z^{4}}\left(\frac{1}{z}-\frac{2}{d}\right), \quad P_{\mathrm{EM}}=\frac{\mathcal{B}^{2} M^{2} Q^{2}}{96 z^{4}} .
$$

Using $\int d t(\cdots)=\int d z / \dot{z}(\cdots)$, we can evaluate the time integral up to some cutoff separation, say $z_{c}$. This gives

$$
\begin{aligned}
\frac{E_{\mathrm{rad}}^{\mathrm{GW}}}{M} & =\mathcal{B}^{5 / 2} M^{7 / 2} \frac{15 d^{2}+24 d z_{c}+32 z_{c}^{2}}{12600\left(d z_{c}\right)^{2}}\left(\frac{1}{z_{c}}-\frac{2}{d}\right)^{3 / 2} \\
& \stackrel{d \rightarrow \infty}{\longrightarrow} \frac{\left(1+\frac{Q^{2}}{M^{2}}\right)^{5 / 2}}{840\left(\frac{z_{c}}{M}\right)^{7 / 2}}
\end{aligned}
$$

and

$$
\begin{aligned}
\frac{E_{\mathrm{rad}}^{\mathrm{EM}}}{M} & =\mathcal{B}^{3 / 2} M^{1 / 2} Q^{2} \frac{3 d^{2}+8 d z_{c}+32 z_{c}^{2}}{360\left(d z_{c}\right)^{2}} \sqrt{\frac{1}{z_{c}}-\frac{2}{d}} \\
& \stackrel{d \rightarrow \infty}{\longrightarrow} \frac{\left(1+\frac{Q^{2}}{M^{2}}\right)^{3 / 2}\left(\frac{Q}{M}\right)^{2}}{120\left(\frac{z_{c}}{M}\right)^{5 / 2}} .
\end{aligned}
$$

Thus, for large initial separations,

$$
\frac{E_{\mathrm{rad}}^{\mathrm{EM}}}{E_{\mathrm{rad}}^{\mathrm{GW}}}=7 \frac{z_{c}}{M} \frac{\left(\frac{Q}{M}\right)^{2}}{1+\frac{Q^{2}}{M^{2}}} .
$$

We can now make some estimates based on the previous formulas in the limit of $d \rightarrow \infty$. These estimates depend on the cutoff scale $z_{c}$. As we will see in the next section, we observe that indeed, for a range of cutoffs around $z_{c} \simeq 1.5 \mathrm{M}$, the analytic approximation captures remarkably well the radiation emission patterns, for both the electromagnetic and the gravitational wave sectors. At the end of the next section we shall make some explicit comparisons between the above formulas and the numerical results. 
TABLE I. Numerical grid structure used (in the notation of Sec. II E of [36]), initial coordinate distance $d / M$, total Arnowitt, Deser, Misner (ADM) mass, charge-to-mass ratio $\pm Q / M$, gravitational $\left(E_{\mathrm{rad}}^{\mathrm{GW}}\right)$ and electromagnetic $\left(E_{\mathrm{rad}}^{\mathrm{EM}}\right)$ radiated energy for our set of simulations. Gravitational radiated energy has been computed using only the $l=2, m=0$ mode, while for the electromagnetic radiated energy only the $l=1, m=0$ multipole was used, as the energy contained in higher-order multipoles is negligible for all configurations.

\begin{tabular}{|c|c|c|c|c|c|c|}
\hline Run & Grid structure & $M_{\mathrm{ADM}}$ & $d / M$ & $|Q| / M$ & $\frac{E_{\mathrm{rad}}^{\mathrm{GW}}}{M_{\mathrm{ADM}}} \times 10^{3}$ & $\frac{E_{\mathrm{rad}}^{\mathrm{EM}}}{M_{\mathrm{ADM}}} \times 10^{3}$ \\
\hline Q+-010_D16_HF64 & $\{(256,128,64,32,16 \times(4,2,1,0.5), M / 64\}$ & 1 & 16 & 0.1 & 0.536 & 0.0426 \\
\hline Q+-020_D16_HF64 & $\{(256,128,64,32,16 \times(4,2,1,0.5), M / 64\}$ & 0.999 & 16 & 0.2 & 0.554 & 0.174 \\
\hline Q+-030_D16_HF64 & $\{(256,128,64,32,16 \times(4,2,1,0.5), M / 64\}$ & 0.997 & 16 & 0.3 & 0.584 & 0.405 \\
\hline Q+-040_D16_HF64 & $\{(256,128,64,32,16 \times(4,2,1,0.5), M / 64\}$ & 0.995 & 16 & 0.4 & 0.627 & 0.754 \\
\hline Q+-050_D16_HF64 & $\{(256,128,64,32,16 \times(4,2,1,0.5), M / 64\}$ & 0.993 & 16 & 0.5 & 0.685 & 1.25 \\
\hline Q+-050_D16_HF80 & $\{(256,128,64,32,16 \times(4,2,1,0.5), M / 80\}$ & 0.993 & 16 & 0.5 & 0.706 & 1.26 \\
\hline Q+-050_D16_HF96 & $\{(256,128,64,32,16 \times(4,2,1,0.5), M / 96\}$ & 0.993 & 16 & 0.5 & 0.714 & 1.26 \\
\hline Q+-060_D16_HF64 & $\{(256,128,64,32,16 \times(4,2,1,0.5), M / 64\}$ & 0.989 & 16 & 0.6 & 0.757 & 1.92 \\
\hline Q+-070_D16_HF64 & $\{(256,128,64,32,16 \times(4,2,1,0.5), M / 64\}$ & 0.985 & 16 & 0.7 & 0.846 & 2.82 \\
\hline Q+-070_D16_HF80 & $\{(256,128,64,32,16 \times(4,2,1,0.5), M / 80\}$ & 0.985 & 16 & 0.7 & 0.875 & 2.84 \\
\hline Q+-070_D16_HF96 & $\{(256,128,64,32,16 \times(4,2,1,0.5), M / 96\}$ & 0.985 & 16 & 0.7 & 0.885 & 2.84 \\
\hline Q+-080_D16_HF64 & $\{(256,128,64,32,16 \times(4,2,1,0.5), M / 64\}$ & 0.981 & 16 & 0.8 & 0.953 & 4 \\
\hline Q+-090_D16_HF64 & $\{(256,128,64,32,16 \times(4,2,1,0.5), M / 64\}$ & 0.976 & 16 & 0.9 & 1.08 & 5.52 \\
\hline Q+-090_D16_HF80 & $\{(256,128,64,32,16 \times(4,2,1,0.5), M / 80\}$ & 0.976 & 16 & 0.9 & 1.12 & 5.58 \\
\hline Q+-090_D16_HF96 & $\{(256,128,64,32,16 \times(4,2,1,0.5), M / 96\}$ & 0.976 & 16 & 0.9 & 1.13 & 5.59 \\
\hline Q+-050_D32_HF96 & $\{(256,176,64,32 \times(8,4,2,1,0.5), M / 96\}$ & 0.996 & 32 & 0.5 & 0.755 & 1.35 \\
\hline Q+-060_D32_HF96 & $\{(256,176,64,32 \times(8,4,2,1,0.5), M / 96\}$ & 0.995 & 32 & 0.6 & 0.84 & 2.08 \\
\hline Q+-070_D32_HF96 & $\{(256,176,64,32 \times(8,4,2,1,0.5), M / 96\}$ & 0.993 & 32 & 0.7 & 0.945 & 3.05 \\
\hline Q+-080_D32_HF96 & $\{(256,176,64,32 \times(8,4,2,1,0.5), M / 96\}$ & 0.99 & 32 & 0.8 & 1.07 & 4.32 \\
\hline Q+-090_D32_HF128 & $\{(256,176,64,32 \times(8,4,2,1,0.5,0.25), M / 128\}$ & 0.988 & 32 & 0.9 & 1.16 & 5.92 \\
\hline Q+-090_D32_HF160 & $\{(256,176,64,32 \times(8,4,2,1,0.5,0.25), M / 160\}$ & 0.988 & 32 & 0.9 & 1.2 & 5.95 \\
\hline Q+-090_D32_HF192 & $\{(256,176,64,32 \times(8,4,2,1,0.5,0.25), M / 192\}$ & 0.988 & 32 & 0.9 & 1.22 & 5.97 \\
\hline Q+-093_D32_HF192 & $\{(256,176,64,32 \times(8,4,2,1,0.5,0.25), M / 192\}$ & 0.987 & 32 & 0.93 & 1.27 & 6.54 \\
\hline Q+-095_D32_HF256 & $\{(256,176,64,32 \times(8,4,2,1,0.5,0.25,0.125), M / 256\}$ & 0.986 & 32 & 0.95 & 1.24 & 6.89 \\
\hline Q+-095_D32_HF320 & $\{(256,176,64,32 \times(8,4,2,1,0.5,0.25,0.125), M / 320\}$ & 0.986 & 32 & 0.95 & 1.29 & 6.94 \\
\hline Q+-097_D32_HF320 & $\{(256,176,64,32 \times(8,4,2,1,0.5,0.25,0.125), M / 320\}$ & 0.986 & 32 & 0.97 & 1.32 & 7.36 \\
\hline Q+-099_D32_HF512 & $\{(256,176,64,32 \times(8,4,2,1,0.5,0.25,0.125,0.0625), M / 512\}$ & 0.985 & 32 & 0.99 & 1.32 & 7.79 \\
\hline Q+-099_D32_HF640 & $\{(256,176,64,32 \times(8,4,2,1,0.5,0.25,0.125,0.0625), M / 640\}$ & 0.985 & 32 & 0.99 & 1.36 & 7.82 \\
\hline Q+-090_D48_HF160 & $\{(256,176,64 \times(16,8,4,2,1,0.5,0.25), M / 160\}$ & 0.992 & 48 & 0.9 & 1.2 & 6.01 \\
\hline
\end{tabular}

\section{NUMERICAL RESULTS}

As in Paper I, we numerically integrate the EinsteinMaxwell system using fourth-order spatial discretization with the LEAN code, which is based on the CACTUS Computational toolkit [27], the CARPET mesh refinement package $[28,29]$ and uses AHFINDERDIRECT for tracking apparent horizons [30,31]. LEAN uses the Baumgarte, Shapiro, Shibata, Nakamura formulation of the Einstein equations $[32,33]$ with the moving puncture method $[34,35]$. We refer the interested reader to Ref. [36] for further details on the numerical methods, and to Paper I for the tests performed with the Einstein-Maxwell implementation.

\section{A. Simulations and convergence properties}

As stated above, we have prepared time-symmetric binary BH puncture-type initial data with $m_{1}=m_{2}=M / 2$, $q_{1}=-q_{2}=Q / 2$, where we vary the charge-to-mass ratio from $Q / M= \pm 0.1$ to $Q / M= \pm 0.99$. Binaries start from rest with initial (coordinate) distance $d / M=16, d / M=32$, or $d / M=48$. These parameters as well as the grid setup and the radiated energy emitted in gravitational $\left(E_{\mathrm{rad}}^{\mathrm{GW}}\right)$ and electromagnetic $\left(E_{\mathrm{rad}}^{\mathrm{EM}}\right)$ waves are listed in Table I. In describing the grid structure, we follow the notation of Sec. II E of [36]: the initial grid consists of $n$ outer levels centered on the origin (remaining static throughout the simulation) and $m$ moving levels with two components centered around each $\mathrm{BH}$; for example, $\{(256,128,64$, $32,16 \times(4,2,1,0.5), M / 64\}$ specifies a grid with five fixed outer components of radii $256,128,64,32$, and 16 , and four moving levels with radii $4,2,1$, and 0.5 . The grid spacing is $h_{f}=M / 64$ on the finer level and successively increases by factors of 2 until the outermost level.

As a test on the correctness of our implementation, we have evolved models Q+-050_D16 and Q+-090_D32 with three different resolutions, as outlined in Table I, and performed a convergence analysis of both the extracted waveforms and the violation of the electric and Einstein constraints.

In Fig. 2, we display the electric and Hamiltonian constraints along the $z$ axis at $t=384 M$ for model Q+090_D32. Each panel displays two curves, one obtained for 

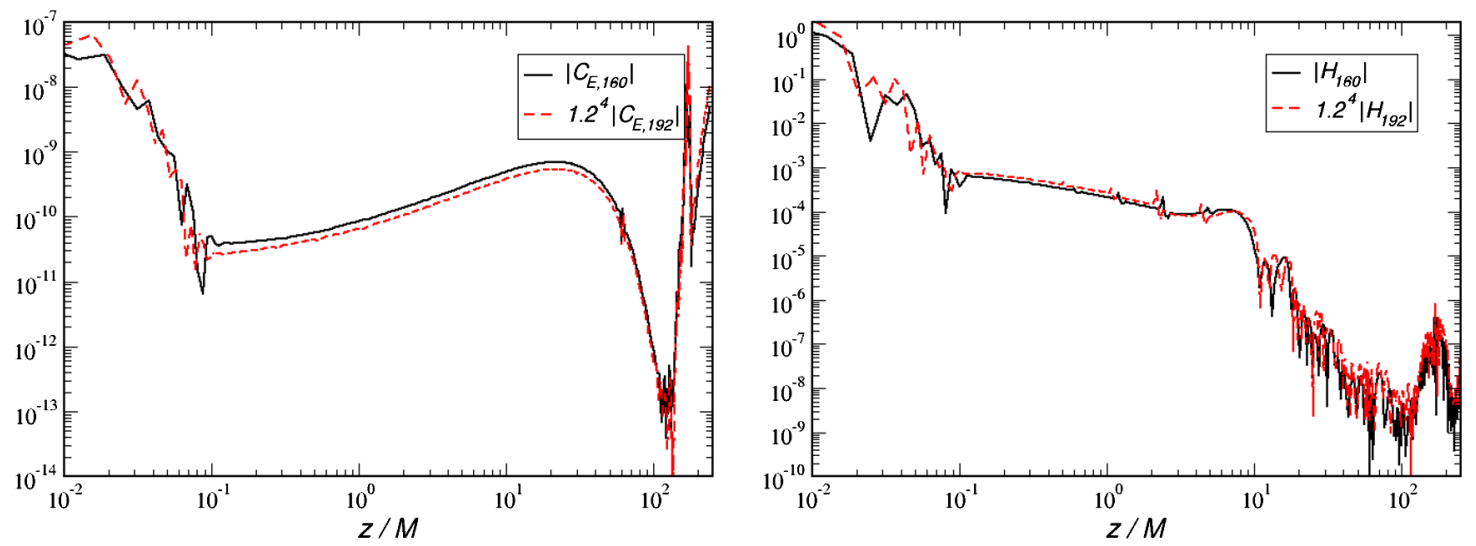

FIG. 2 (color online). The electric (left panel) and Hamiltonian (right panel) constraints along the collision axis at time $t=384 M$ for model Q+-090_D32. The solid (black) curves display the result obtained for lower resolution $h_{f}=M / 160$ and the dashed (red) curves show that obtained for higher resolution $h_{f}=M / 196$ and amplified by $1.2^{4}$ for the expected fourth-order convergence.

a resolution $h_{f}=M / 160$ on the finest grid and one obtained for a higher resolution $h_{f}=M / 192$ for which the result has been amplified by the expected convergence factor $1.2^{4}$. Even though some constraint violations are generated by the outer boundary treatment, the overall violations are small (as compared with the magnitude of the individual terms summed over in the constraints) and display fourth-order convergence. For the momentum constraints we observe similar behavior to that of the Hamiltonian constraint.

We have complemented these two simulations with an additional one using $h_{f}=M / 128$ in order to estimate the uncertainties in the energy radiated in gravitational and electromagnetic waves. We obtain for the electromagnetic energy $E_{\text {rad }}^{\mathrm{EM}}$ the values $0.5865 \% M, 0.5883 \% M$, and $0.5888 \% M$, respectively, for $h_{f}=M / 128,1 / 160$, and $1 / 192$, which is in good agreement with fourth-order convergence and gives a relative error of half a percent or better for $h_{f} \leq M / 128$. Likewise, we obtain for the energy emitted in gravitational waves $E_{\mathrm{rad}}^{\mathrm{GW}}=0.1189 \% M$, $0.1214 \% M$, and $0.1222 \% M$, again in good agreement with fourth-order convergence, and yielding a relative error of about $3 \%$ for $h_{f}=M / 128$ and $1.5 \%$ or less for $h_{f} \leq M / 160$. We obtain similar error estimates for the model Q+-050_D16 using the three resolutions $h_{f}=M / 64$, $h_{f}=M / 80$, and $h_{f}=M / 96$. This observation confirms our expectation that accurate evolutions with larger magnitudes of the electric charge require higher numerical resolution. We also monitored the uncertainties in the radiated energies arising from extraction at finite radius by extrapolating the results to infinity. For this purpose, we have extracted the wave signals at $R_{\mathrm{ex}} / M=80,100,110$, $120,130,140,150$ and 160 . By extrapolating the values obtained at these finite radii to infinity using a $a_{0}+a_{1} / R_{\mathrm{ex}}$ dependence, we determine the relative uncertainties at $R_{e x}=160 M$ to be about $0.5 \%$ for the electromagnetic and $1 \%$ for the gravitational wave energy radiated away from the binary. Unless stated otherwise, the reported energies refer to the extrapolated values. A further uncertainty in our results arises from the finite initial separation of the BHs. We estimate the resulting error by studying collisions for $Q / M= \pm 0.9$ starting from separations $d / M=16,32$, and 48 . As intuitively expected, the radiated energies mildly increase with initial separation. By extrapolating the results to infinite $d$ assuming a $a_{0}+a_{1} / d$ dependence, we obtain a numerical uncertainty of about $5 \%$ for our values obtained for $d / M=32$. Combining all three sources of errors, we estimate the total uncertainty to be $6 \%$ for $E_{\mathrm{rad}}^{\mathrm{EM}}$ and $7.5 \%$ for $E_{\mathrm{rad}}^{\mathrm{GW}}$. If interpreted as energy radiated by head-on collisions starting at finite separation, these uncertainty estimates drop by a factor of about 3 .

\section{B. Waveforms and integrated energy}

In Fig. 3 we display the waveforms $\psi_{4}^{20}$ and $\phi_{2}^{10}$ obtained at finite extraction radius for a representative subset of the initial configurations listed in Table I.

Note that the configurations studied in this work differ qualitatively from those of Paper I in the final outcome of the merger: a charged $\mathrm{BH}$ in Paper I but an electrically neutral, i.e., a Schwarzschild $\mathrm{BH}$, in this study. In consequence, the ringdown of the collisions in Paper I exhibits a superposition of both gravitational and electromagnetic quasinormal modes (QNM) in both the $\psi_{4}^{20}$ and $\phi_{2}^{10}$ waveforms. For the electrically neutral postmerger BHs of this study, in contrast, the gravitational wave signal $\psi_{4}^{20}$ matches the ringdown of the neutral Schwarzschild $\mathrm{BH}$, and we find a strong electromagnetic QNM component in $\phi_{2}^{10}$. We find no signs of mixing between electromagnetic and gravitational modes. For instance, for all configurations of Table I, the $\phi_{2}^{10}$ waveform is very well described by the lowest electromagnetic ringdown mode [37,38]. In fact, one can recover the lowest electromagnetic QNM of Schwarzschild BHs, as given by perturbative studies, with an accuracy of $0.5 \%$. 

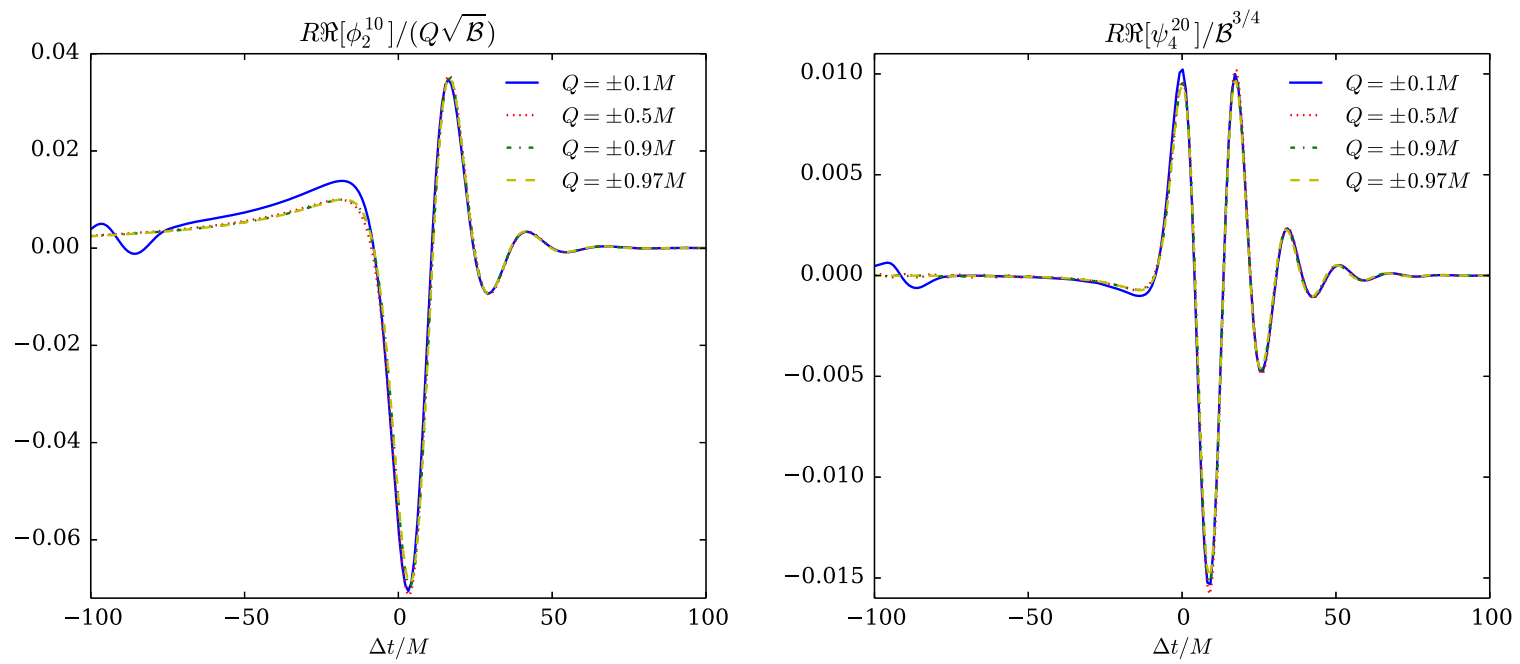

FIG. 3 (color online). Real part of the electromagnetic $(l=1, m=0$ mode $)$ and gravitational $(l=2, m=0$ mode $)$ waveforms. These have been conveniently rescaled and shifted in time so that their peaks coincide.

We have further found that the dependency of the multipoles $\psi_{4}^{20}$ and $\phi_{2}^{10}$ on the parameters $\mathcal{B}$ and $Q$ is very well modeled by the following simple scaling laws: $\psi_{4}^{20} \sim$ $\mathcal{B}^{3 / 4}$ and $\phi_{2}^{10} \sim Q \sqrt{\mathcal{B}}$. For the oppositely charged binaries of the present study, this scaling appears to be satisfied with an even higher accuracy than in the equal-charge case displayed in Fig. 5 of Paper I. Possibly this is a consequence of the vanishing charge of the final $\mathrm{BH}$. We indeed observe that most of the waveform signal is emitted after the formation of the common apparent horizon and would therefore be expected to carry the signature of the final $\mathrm{BH}$. While the ringdown frequency is determined by the quasinormal ringing of a neutral $\mathrm{BH}$, it is interesting to note that the amplitude can be recovered using the above scaling laws. In consequence, the knowledge of the $\psi_{4}^{20}$ and $\phi_{2}^{10}$ multipoles for a single charge-to-mass ratio allows us to derive by rescaling the corresponding waveforms for any other charge-to-mass ratio without the need of performing any other numerical evolution.

We compute the total radiated energies according to Eqs. (2.22) and (2.23). To account for spurious, unphysical radiation resulting from the initial data construction, we start the integration of the radiated fluxes at some finite time $\Delta t$ after the start of the simulation, thus allowing the spurious pulse to first radiate off the computational domain. In practice, we find a value $\Delta t=R_{\mathrm{ex}}+50 M$ to be sufficient for this purpose. The radiated energies thus obtained are plotted in Fig. 4 as functions of the chargeto-mass ratio and quantitatively illustrate the scaling discussed in the previous paragraph.

These results contrast with the corresponding equal charge collisions of Paper I, where the emitted gravitational radiation decreases with increasing charge because of its decelerating effect and the correspondingly low collision velocities, and the emitted electromagnetic radiation peaks at around $Q / M=0.6$. In the case of opposite charges, in contrast, both gravitational and electromagnetic radiation increase with $Q / M$, and the electromagnetic radiation becomes the dominating channel for $|Q| / M \gtrsim 0.37$.

As already mentioned at the end of the last section, we observe a good agreement between our simple analytic model of Sec. III and the numerical simulations we have just presented, for a range of cutoffs for the former. For instance, setting $z_{c}=1.5 \mathrm{M}$, we make the following observations: (i) For the configuration $|Q| / M=0.99$, the ratio of energy in electromagnetic to gravitational radiation

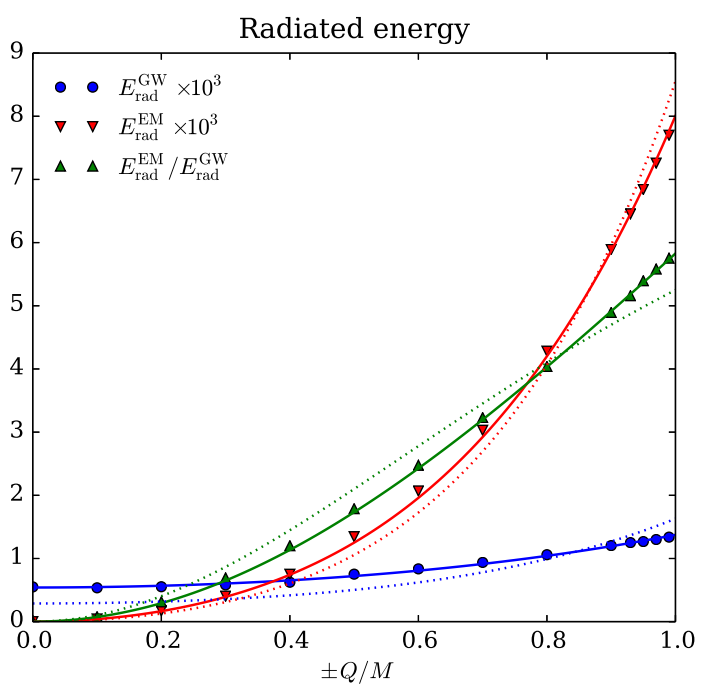

FIG. 4 (color online). Total energy radiated in the electromagnetic $\left(E_{\mathrm{rad}}^{\mathrm{EM}}\right)$ and gravitational $\left(E_{\mathrm{rad}}^{\mathrm{GW}}\right)$ channels. Solid lines show a fit to the numerical results of the form $E_{\mathrm{rad}}^{\mathrm{GW}}=8.53 \times$ $10^{-5}+4.55 \times 10^{-4} \mathcal{B}^{3 / 2}, E_{\mathrm{rad}}^{\mathrm{EM}}=4.00 \times 10^{-3} Q^{2} \mathcal{B}$, in agreement with the scaling used in Fig. 3. Dotted lines show results from the analytic approximation taking $z_{c} / M=1.5$. 
obtained in our numerical simulations is $\sim 5.8$ (cf. Table I), whereas that obtained from our simple analytic approximation is 5.2; cf. Eq. (3.15). (ii) Equal amounts of electromagnetic and gravitational radiated energies are obtained for $|Q| / M \sim 0.37$ in the numerical simulations and $|Q| / M=0.31$ in the analytic model. The analytical results thus reproduce the numerical values with an error between $10 \%$ and $20 \%$. A comparison of the energies emitted in gravitational and electromagnetic radiation for the entire range $Q / M$ is shown in Fig. 4. Even though a discrepancy at a level of about $10 \%$ is visible, the analytic prediction captures the main features of the energy emission remarkably well.

Cutoff independent estimates are provided by the ratio of the energy emitted in either gravitational or electromagnetic waves for two different values of the charge. For instance, from the numerical simulations

$$
\frac{E_{\mathrm{rad}}^{\mathrm{GW}}(|Q|=0.99)}{E_{\mathrm{rad}}^{\mathrm{GW}}(Q=0)} \sim 2.7, \quad \frac{E_{\mathrm{rad}}^{\mathrm{EM}}(|Q|=0.99)}{E_{\mathrm{rad}}^{\mathrm{EM}}(Q=0.1)} \sim 184,
$$

whereas the corresponding values from the analytical approximation are, from (3.13) and (3.14),

$$
\frac{E_{\mathrm{rad}}^{\mathrm{GW}}(|Q|=0.99)}{E_{\mathrm{rad}}^{\mathrm{GW}}(Q=0)} \sim 5.6, \quad \frac{E_{\mathrm{rad}}^{\mathrm{EM}}(|Q|=0.99)}{E_{\mathrm{rad}}^{\mathrm{EM}}(Q=0.1)} \sim 269,
$$

corresponding to mismatches of $\sim 2$ and $\sim 1.5$, respectively.

\section{CONCLUSIONS}

The number of applications of numerical relativity to high energy physics has been growing enormously in recent years [39]. One particular line of research in this area has been the understanding of high energy collisions of BHs $[6,11,40,41]$ and other compact objects [12,14], and a main open question in this context concerns the impact of electric charge on the collision dynamics. To address this question we have continued in this paper the program initiated in Paper I of studying charged $\mathrm{BH}$ collisions. We have here focused on oppositely charged BHs with the same mass-as to maximize the acceleration of the system and hence the gravitational and electromagnetic wave emission - and have shown that the numerical simulations and the extraction of the observable quantities - gravitational and electromagnetic radiation- are well under control.

We have successfully evolved configurations with $|Q| / M$ ranging from 0.1 to 0.99 , once again showing that cases with nearly extremal charge, albeit requiring higher numerical resolution, are simpler to model numerically than the corresponding nearly extremal spin cases $[42,43]$.

The observed qualitative behavior of the energy radiated away is summarized in Fig. 4 and demonstrates that both electromagnetic and gravitational radiation increase monotonically as the (opposite) charges are increased. More surprisingly, our study has revealed a simple, apparently universal scaling of the energy dependence on the charge magnitude that can be seen both in Fig. 4 and in the waveforms presented in Fig. 3. This scaling suggests that the head-on collision of charged BHs with opposite charge to mass ratios may have a (hidden) conformal symmetry, a possibility deserving further study. We have further shown that the radiation emission is well described by a simple analytical model of two nonrelativistic charges in Minkowski space. The radiation emission predicted for the gravitational quadrupole and electromagnetic dipole time variations by numerical relativity calculations and by analytic methods show good agreement in Fig. 4.

There are two natural extensions of this study. One is to perform high energy collisions of charged BHs. Introducing nonzero boosts into the initial data, however, represents a nontrivial challenge since the full (coupled) system of constraint equations needs to be solved. Work in this direction is underway.

Non-head-on collisions or binaries in quasicircular orbits are another natural extension of our results. This problem is understood for neutral, spinning binaries, where it was observed that the total radiation output in the process increases for larger final black hole spins. In simple terms, this is because the innermost stable circular orbit (ISCO) moves inward and the binary can sweep higher frequencies and radiate more strongly. This observation can be naturally accounted for by noticing that the spin of the final black hole is determined by the intrinsic and orbital angular momentum of point particles at the ISCO [44]. Thus, particles with spins aligned have a stronger impact in the final black hole spin. For charged particles a similar reasoning applies; namely, since the ISCO of charged particles moves inward (and the ISCO frequency increases) when charge is added to black holes, quasicircular inspirals of equal-charge binaries would give rise to larger energy fluxes than opposite-charged ones. Such scenarios would then provide ideal prospects for maximizing the gravitational energy output from the system.

\section{ACKNOWLEDGMENTS}

We thank J. C. Degollado for helpful discussions. M. Z. is supported by NSF Grants No. OCI-0832606, No. PHY0969855, No. AST-1028087, and No. PHY-1229173. V. C. acknowledges financial support provided under the European Union's FP7 ERC Starting Grant "The dynamics of black holes: testing the limits of Einstein's theory" Grant Agreement No. DyBHo-256667. U. S. acknowledges support by the FP7-PEOPLE-2011-CIG CBHEO Grant No. 293412, the STFC Grant No. ST/I002006/1, the XSEDE Grant No. PHY-090003 by the National Science Foundation, the COSMOS supercomputer infrastructure, part of the DiRAC HPC Facility funded by STFC and BIS, 
and the Centro de Supercomputacion de Galicia (CESGA) under Grant No. ICTS-2013-249, This research was supported in part by Perimeter Institute for Theoretical Physics. Research at Perimeter Institute is supported by the Government of Canada through Industry Canada and by the Province of Ontario through the Ministry of Economic Development \& Innovation. This work was supported by the
NRHEP 295189 FP7-PEOPLE-2011-IRSES Grant, and by FCT-Portugal through Projects No. PTDC/FIS/116625/ 2010 and No. CERN/FP/123593/2011. Computations were performed on the "Baltasar Sete-Sois" cluster at IST, the "Blafis" cluster at Universidade de Aveiro, the NICS Kraken Cluster, the SDSC Trestles Cluster, Cambridge's COSMOS, on the "venus" cluster at YITP, and CESGA's Finis Terrae.
[1] F. Pretorius, arXiv:0710.1338.

[2] S. W. Hawking, Phys. Rev. Lett. 26, 1344 (1971).

[3] R. Penrose (unpublished).

[4] D. M. Eardley and S. B. Giddings, Phys. Rev. D 66, 044011 (2002).

[5] H. Witek, M. Zilhão, L. Gualtieri, V. Cardoso, C. Herdeiro, A. Nerozzi, and U. Sperhake, Phys. Rev. D 82, 104014 (2010).

[6] U. Sperhake, V. Cardoso, F. Pretorius, E. Berti, and J. A. Gonzalez, Phys. Rev. Lett. 101, 161101 (2008).

[7] M. Campanelli, C. Lousto, and Y. Zlochower, Phys. Rev. D 74, 084023 (2006).

[8] D.-I. Choi, B. J. Kelly, W. D. Boggs, J. G. Baker, J. Centrella, and J. van Meter, Phys. Rev. D 76, 104026 (2007).

[9] M. Campanelli, C. Lousto, and Y. Zlochower, Phys. Rev. D 74, 041501 (2006).

[10] D. A. Hemberger, G. Lovelace, T. J. Loredo, L. E. Kidder, M. A. Scheel, B. Szilágyi, N. W. Taylor, and S. A. Teukolsky, Phys. Rev. D 88, 064014 (2013).

[11] U. Sperhake, E. Berti, V. Cardoso, and F. Pretorius, Phys. Rev. Lett. 111, 041101 (2013).

[12] M. W. Choptuik and F. Pretorius, Phys. Rev. Lett. 104, 111101 (2010).

[13] L. Rezzolla and K. Takami, Classical Quantum Gravity 30, 012001 (2013).

[14] W. E. East and F. Pretorius, Phys. Rev. Lett. 110, 101101 (2013).

[15] C. Herdeiro, M. O. Sampaio, and C. Rebelo, J. High Energy Phys. 07 (2011) 121.

[16] F. S. Coelho, C. Herdeiro, and M. O. Sampaio, Phys. Rev. Lett. 108, 181102 (2012).

[17] R. M. Wald, Phys. Rev. D 6, 406 (1972).

[18] M. Zilhão, V. Cardoso, C. Herdeiro, L. Lehner, and U. Sperhake, Phys. Rev. D 85, 124062 (2012).

[19] M. Ansorg, B. Bruegmann, and W. Tichy, Phys. Rev. D 70, 064011 (2004).

[20] S. Komissarov, Mon. Not. R. Astron. Soc. 382, 995 (2007).

[21] C. Palenzuela, L. Lehner, O. Reula, and L. Rezzolla, Mon. Not. R. Astron. Soc. 394, 1727 (2009).
[22] D. R. Brill and R. W. Lindquist, Phys. Rev. 131, 471 (1963).

[23] M. Alcubierre, J. C. Degollado, and M. Salgado, Phys. Rev. D 80, 104022 (2009).

[24] M. Zilhão, M. Ansorg, V. Cardoso, L. Gualtieri, C. Herdeiro, U. Sperhake, and H. Witek, Phys. Rev. D 84, 084039 (2011).

[25] E. Newman and R. Penrose, J. Math. Phys. (N.Y.) 3, 566 (1962).

[26] J. D. Jackson, Classical Electrodynamics (Wiley, New York, 1998), 3rd ed.

[27] Cactus Computational Toolkit, http://www.cactuscode.org/.

[28] E. Schnetter, S. H. Hawley, and I. Hawke, Classical Quantum Gravity 21, 1465 (2004).

[29] Mesh Refinement with Carpet, http://www.carpetcode.org/.

[30] J. Thornburg, Classical Quantum Gravity 21, 743 (2004).

[31] J. Thornburg, Phys. Rev. D 54, 4899 (1996).

[32] M. Shibata and T. Nakamura, Phys. Rev. D 52, 5428 (1995).

[33] T. W. Baumgarte and S. L. Shapiro, Phys. Rev. D 59, 024007 (1998).

[34] M. Campanelli, C. Lousto, P. Marronetti, and Y. Zlochower, Phys. Rev. Lett. 96, 111101 (2006).

[35] J. G. Baker, J. Centrella, D.-I. Choi, M. Koppitz, and J. van Meter, Phys. Rev. Lett. 96, 111102 (2006).

[36] U. Sperhake, Phys. Rev. D 76, 104015 (2007).

[37] E. Berti, V. Cardoso, and C. M. Will, Phys. Rev. D 73, 064030 (2006).

[38] E. Berti, V. Cardoso, and A. O. Starinets, Classical Quantum Gravity 26, 163001 (2009).

[39] V. Cardoso et al., Classical Quantum Gravity 29, 244001 (2012).

[40] M. Shibata, H. Okawa, and T. Yamamoto, Phys. Rev. D 78, 101501 (2008).

[41] U. Sperhake, E. Berti, V. Cardoso, F. Pretorius, and N. Yunes, Phys. Rev. D 83, 024037 (2011).

[42] G. Lovelace, M. Boyle, M. A. Scheel, and B. Szilagyi, Classical Quantum Gravity 29, 045003 (2012).

[43] C. O. Lousto, H. Nakano, Y. Zlochower, B. C. Mundim, and M. Campanelli, Phys. Rev. D 85, 124013 (2012).

[44] A. Buonanno, L. E. Kidder, and L. Lehner, Phys. Rev. D 77, 026004 (2008). 